\title{
Guidelines
}

\section{The health of prisoners: summary of NICE guidance}

Richard Bradshaw, ${ }^{1}$ guideline chair, NICE Physical Health of People in Prison Guideline Ben A J Pordes, ${ }^{2}$ senior project manager

Hannah Trippier, ${ }^{2}$ senior research fellow

Nick Kosky, ${ }^{3}$ guideline chair, NICE Mental health of adults in contact with the criminal justice system guideline

Steve Pilling, ${ }^{4}$ clinical advisor

Fionnuala O'Brien, project manager

On behalf of the Guideline Committee for the NICE guideline on physical health of people in prisons and the NICE guideline on mental health of adults in contact with the criminal justice system

\section{${ }^{1}$ Former Director of Offender Health Department of Health / National Offender Management Service}

2 National Guideline Centre, 180 Great Portland Street, London W1W 5QZ

${ }^{3}$ Dorset Healthcare University Foundation Trust, Forston Clinic

${ }^{4}$ National Guideline Alliance, Royal College of Obstetricians and Gynaecologists, London, NW1

Correspondence to: N Kosky Nick.Kosky@dhuft.nhs.uk?

\section{Box Start}

What you need to know

- People in custodial settings are entitled to the same level and quality of physical and mental health services as NHS patients in the community without restrictions

- Perform comprehensive extended physical and mental health assessment on entry into and release from custodial settings

- Monitor and treat chronic health conditions for those serving custodial sentences, offer testing for infectious diseases, and explore the possibility of mental health conditions

- The prevalence of mental health problems among people in prison is about 10 times higher than in the general population

- On a person's release from a custodial sentence, primary care services should receive a structured handover of new and ongoing health problems, which can guide them as they reassume responsibility for coordinating care 
Compared with the general population, adults in contact with the criminal justice system have higher rates of mental and physical health problems. Such people include those under arrest, in prison, on license, or serving a community sentence. Most live in the community or serve short custodial sentences[1], and will feature on the patient list of most general practices. Care for these people should be equivalent to that of people without a history of contact with the criminal justice system. There is little to suggest that, for most people, treatments that are effective in a non-criminal justice setting are not effective in the criminal justice setting.

Some of the most prevalent problems include substance misuse, personality disorder, depression, anxiety, respiratory disease, diabetes, and other chronic health conditions. Being held in prison is associated with increased suicide rates and high levels of mental health problems. People in contact with the criminal justice system should have the same access to NHS treatment as the general population at all stages. Lack of access to healthcare can exacerbate existing problems and affect reintegration to society and reduction of reoffending. Good health helps increase stability in interpersonal relationships and finding a job or meaningful occupation, and so results in greater social stability. These factors reduce the likelihood of reoffending. Missed opportunities to manage health conditions while someone is in custody increases the risk of deterioration and requiring a higher level of treatment input once the person is discharged.

This article summarises the most recent recommendations from the National Institute of Health and Care Excellent (NICE) on the identification and treatment of physical health in prisons and mental health in the criminal justice system.[2] [3]

\section{Recommendations}

NICE recommendations are based on systematic reviews of best available evidence and explicit consideration of cost effectiveness. When minimal evidence is available, recommendations are based on the Guideline Committee's experience and opinion of what constitutes good practice. Evidence levels for the recommendations are given in italic in square brackets.

\section{Assessment and identification of active, chronic, or undiagnosed problems}

In prison the first stage health assessment is a general health screen for health issues that will be relevant during the person's initial imprisonment (box 1). This process is to ensure that an assessment and first management steps of key health and lifestyle factors is done at 
first reception into prison. This allows for early identification of health risks and needs to be identified and where relevant referrals need to be made.

\section{Box Start}

\section{Box 1. First stage health assessment}

Prison sentence

1. Has the person committed murder, manslaughter or another offence with a long sentence?

Prescribed medicines

2. Is the person taking any prescribed medicines (for example, insulin) or over-the-counter medicines (such as creams or drops)? If so:

- what are they

- what are they for

- how do they take them?

Physical injuries

3. Has the person received any physical injuries over the past few days, and if so:

- what were they

- how were they treated?

\section{Other health conditions}

4. Does the person have any of the following:

- allergies, asthma, diabetes, epilepsy or history of seizures

- chest pain, heart disease

- chronic obstructive pulmonary disease

- tuberculosis, sickle cell disease

- hepatitis B or C virus, HIV, other sexually transmitted infections

- learning disabilities

- neurodevelopmental disorders

- physical disabilities?

5. Are there any other health problems the person is aware of that have not been reported?

6. Are there any other concerns about the person's health?

\section{Additional questions for women}

7. Does the woman have reason to think she is pregnant, or would she like a pregnancy test?

Living arrangements, mobility and diet

8. Does the person need help to live independently?

9. Do they use any equipment or aids (for example, walking stick, hearing aid, glasses, dentures, continence aids or stoma)?

10. Do they need a special medical diet?

Past or future medical appointments

11. Has the person seen a doctor or other healthcare professional in the past few months? If so, what this was for?

12. Does the person have any outstanding medical appointments? If so, who are they with, and when?

Alcohol and substance misuse

13. Does the person drink alcohol, and if so:

- how much do they normally drink?

- how much did they drink in the week before coming into custody? 
14. Has the person used street drugs in the last month? If so, how frequently? When did they last use:

- heroin

- methadone

- benzodiazepines

- amphetamine

- cocaine or crack

- novel psychoactive substances

- cannabis

- anabolic steroids

- performance and image enhancing drugs?

Problematic use of prescription medicines

15. Has the person used prescription or over-the-counter medicines in the past month:

- that were not prescribed or recommended for them or

- for purposes or at doses that were not prescribed?

- If so, what was the medicine and how did they use it (frequency and dose)?

\section{Mental health}

16. Has the person ever seen a healthcare professional or service about a mental health problem (including a psychiatrist, GP, psychologist, counsellor, community mental health services, alcohol or substance misuse services or learning disability services)?

If so, who did they see and what was the nature of the problem?

17. Has the person ever been admitted to a psychiatric hospital, and if so:

- when was their most recent discharge

- what is the name of the hospital

- what is the name of their consultant?

18. Has the person ever been prescribed medicine for any mental health problems? If so:

- what was the medicine

- when did they receive it

- when did they take the last dose

- what is the current dose (if they are still taking it)

- when did they stop taking it?

Self-harm and suicide risk

19. Is the person:

- feeling hopeless or

- currently thinking about or planning to harm themselves or attempt suicide?

20. Has the person ever tried to harm themselves, and if so:

- do they have a history of suicide attempts

- was this inside or outside prison

- when was the most recent incident

- what was the most serious incident? 
- At first reception into prison, a healthcare professional (or trained healthcare assistant under the supervision of a registered nurse) should carry out a health assessment for every person. Do this before the person is allocated to a cell. As part of the assessment, identify:

- Any issues that may affect the person's immediate health and safety before the second stage health assessment

- Priority health needs to be addressed at the next clinical opportunity.

[Based on low to very low quality evidence from diagnostic accuracy studies, health economic analysis and Guideline Committee (GC) consensus]

- The first stage health assessment [see Box 1] should include questions covering:

- Physical health

- Alcohol use

- Substance misuse

- Mental health

- Self harm and suicide risk.

[Based on low to very low quality evidence from diagnostic accuracy studies, and GC consensus]

- Carry out a medicines reconciliation (in line with NICE's guideline on medicines optimisation[4]) before the second stage health assessment. [Based on moderate to low quality evidence from randomised controlled trials, NICE medicines optimisation guidance 2015, and GC consensus]

- A healthcare professional (such as a registered general nurse) should carry out a second stage health assessment for every person in prison. Do this within seven days of the first stage health assessment, and include as a minimum the points in box 2. [Based on very low quality evidence from a diagnostic accuracy study and GC consensus]

\section{Box Start}

\section{Box 2: Second stage health assessment (within seven days of first stage health assessment)}

- Review the actions and outcomes from the first stage health assessment

- Measure and record the person's height, weight, pulse, blood pressure, and temperature, and carry out a urine analysis

\section{Ask the person about}

- Any previous misuse of alcohol, use of drugs, or improper use of prescription medicine

- If they have ever had a head injury or lost consciousness, and if so:

- How many times this has happened

- Whether they have ever been unconscious for more than 20 minutes

- Whether they have any problems with memory or concentration

- Smoking history

- Date of their last sexual health screen

- Any history of serious illness in their family (for example, heart disease, diabetes, epilepsy, cancer, or chronic conditions)

- Their expected release date, and if $<1$ month plan a pre-release health assessment

- Whether they have ever had a screening test (for example, a cervical screening test or mammogram)

- Whether they have, or have had, any gynaecological problems 


\section{Box End}

\section{Chronic health conditions}

Health assessment for individuals in the community (that is, on probation or license or released from prison) does not have a formally set out process akin to the first and second stage assessments in prison. However, the NICE guidelines advise professionals on what to include in an assessment and emphasise the importance of performing mental health assessments and of reviewing any available records.

- All practitioners carrying out a mental health assessment for people in contact with the criminal justice system should take into account the following:

- The nature and severity of presenting mental health problems (including cognitive functioning) and their development and history

- Coexisting mental health problems

- Coexisting substance misuse problems (including novel psychoactive substances)

- Coexisting physical health problems

- Social and personal circumstances (including personal experience of trauma)

- Social care, educational, and occupational needs

- People's strengths

- Available support networks (and a person's capacity to make use of them)

- Previous care, support, and treatment, including how the person responded to these

- Offending history and how this may interact with mental health problems.

[Based on the experience and opinion of the GC using a formal consensus method]

\section{Communication, coordination, and care planning}

As with the general population, primary care services lead on coordination of care for those in the criminal justice system who are not serving custodial sentences, and this can follow usual processes. Primary care services should receive and share information required for the effective treatment of health conditions from and with healthcare staff caring for people within the criminal justice system.

- Develop a mental health plan of care in collaboration with the person and, when possible, their family, carers, and advocates. All practitioners developing the plan should ensure it is integrated with care plans from other services and includes:

- A profile of the person's needs (including physical health needs), identifying agreed goals and the means to progress towards goals

- Identification of the roles and responsibilities of those practitioners involved in delivering the plan

- The implications of any mandated treatment programmes, post-release licences, and transfer between institutions or agencies, in particular release from prison

- A clear strategy to access all identified interventions and services

- Agreed outcome measures and timescale to evaluate and review the plan

- A risk management and a crisis plan if developed 
- An agreed process for communicating the plan (such as the Care Programme Approach or Care Treatment Plan) to all relevant agencies, the person, and their families and carers.

\section{[Based on the experience and opinion of the GC using a formal consensus method]}

- Carry out an individual risk assessment to determine if the person can hold his or her medicines in possession. Allow people in prison to hold all medicine in possession unless they do not pass the risk assessment (see box 3 for more information). [Based on moderate to low quality evidence from randomised controlled trials, and low to very low quality evidence from qualitative interview and focus group studies with people in prisons or young offender institutions and health professionals and other staff working in prisons or young offender institutions]

\section{Box Start}

\section{Box 3: Service level recommendation}

Commissioners and providers of criminal justice services and healthcare services should ensure effective identification, assessment, coordination, and delivery of care for all people, including those who are transferring from young offender services, with a mental health problem in contact with the criminal justice system (including probation service providers). In particular, ensure that:

- All people with a severe or complex mental health problem have a designated care coordinator

- During transitions between services, care plans are shared and agreed between all services

- Effective protocols are in place to support routine data sharing and, when necessary, joint plans of care between health services (including primary and secondary care services) and criminal justice agencies to reduce unnecessary assessments and promote effective interventions

[Based on the experience and opinion of the $G C$ ]

Box End

\section{Planning for release and transition}

Transition between community and custody is a time where there is an increased risk of disruptions to treatment and a deterioration in health. Custodial healthcare teams need to keep community based primary care services involved throughout the process of planning for release or during transition, including sharing a care summary and post-release action plan.

On release from custody, responsibility for care will return to the person's primary care service. As with the general population, care planning and coordination require the input from a combination of health and social care services. What may be unique to supporting adults in contact with the criminal justice system is the nature of multidisciplinary collaborations across health, social care, and criminal justice services to address the care and management required.

- Carry out a pre-release health assessment for any person with complex needs. This should be led by prison primary healthcare and involve multidisciplinary team members and the person. It should take place at least one month before the date the person is expected to be released. [Based on low to very low quality evidence from qualitative interview and focus group studies with people in prisons or young offender institutions, and health 
professionals and other staff working in prisons or young offender institutions, and GC consensus]

- Include the following in the care summary and post-release action plan for all people:

- Any significant health events that affected the person while in prison, for example:

$\circ$ New diagnoses

$\circ$ Hospital admissions

$\circ$ Instances of self harm

- Any health or social care provided in prison

- Details of any ongoing health and social care needs, including:

$\circ$ Medicines being taken

o Mental health or substance misuse

- Future health and social care appointments, including appointments with:

$\circ$ Secondary and tertiary care

$\circ$ Mental health services

- Substance misuse and recovery services

○ Social services.

[Based on low to very low quality evidence from qualitative interview and focus group studies with people in prisons or young offender institutions, and health professionals and other staff working in prisons or young offender institutions, and $G C$ consensus]

- Give the person a copy of the care summary and post-release plan. [Based on GC consensus]

- Help people who are being released from prison to find and register with a community GP if they were not previously registered with one. [Based on low to very low quality evidence from qualitative interview and focus group studies with people in prisons or young offender institutions, and health professionals and other staff working in prisons or young offender institutions, and GC consensus]

- Before the person is released, liaise with services that will be providing care and support after the person leaves prison. Liaison should include (as needed):

- Primary care

- Secondary and tertiary specialist services (such as for HIV infection, tuberculosis, cancer)

- Mental health or learning disability services

- Substance misuse services

- National Probation Service

- Community rehabilitation company

- Social services

- Family or carers

- External agencies such as home care.

[Based on low to very low quality evidence from qualitative interview and focus group studies with people in prisons or young offender institutions, and health professionals and other staff working in prisons or young offender institutions, and GC consensus]

\section{Implementation and overcoming barriers}


There are specific challenges to delivering healthcare to this population:

- Personal factors

- Poor history of engaging with healthcare due to chaotic lifestyle and a lack of trust in services.

- Staff factors

- Challenge of managing behaviours associated with disorders such as attention deficit hyperactivity disorder and autism spectrum disorders, acquired cognitive impairment, and chronic drug and alcohol misuse within an offending context

- Lack of effective training for staff in the identification and support of health needs and complexity

- Lack of understanding of how the criminal justice system works.

- Organisational factors

- Difficulty in effective interagency cooperation across boundaries-for example, geographical boundaries in arranging transfer from one region to another or information sharing policies between different criminal justice services.

\section{Box Start}

\section{Future research}

- Assessment and identification-When should subsequent health assessments be carried out in prison for people serving long term sentences?

- Communication, coordination, and care planning-What models for the coordination and delivery of care for people in contact with the criminal justice provide for the most effective and efficient coordination of care and improve access and uptake of services?

- Chronic health conditions -What is the prevalence of disease in the UK prison population?

- Access to medicines - Does the use of directly observed supply of named high risk medicines (that is, not supplying the medicines to people to hold in possession), reduce diversion, misuse, and non-adherence?

\section{Box End}

\section{Box Start}

\section{Guideline into practice}

- For those working in a non-custodial setting, to what extent are you aware of the organisation and process of healthcare for adults in contact with the criminal justice system?

- What actions could you take in your consultations or in your organisation to better support care for those in contact with the criminal justice system?

\section{Box End}

\section{Box Start}

\section{How patients were involved in the creation of this article}

No patients with experience of care in the criminal justice system were involved in the creation of this summary. Committee members involved in this guideline included lay members who had direct experience of being involved with the criminal justice system and who contributed to the formulation of the recommendations summarised here.

Box End

\section{Box Start}

Methods 
The mental health of adults in contact with the criminal justice system guideline was produced by the National Guideline Alliance. The Guideline Committee (GC) comprised two forensic psychiatrists, two psychologists, a probation officer, two senior managers from a prison setting, a substance misuse practitioner/psychologist, a legal services representative, a transitions expert, a nurse, a primary care representative, a carer, two service users, a police officer, and a prison officer.

The physical health of people in prison guideline was developed by the National Guideline Centre. The GC for this guideline comprised a former director of Offender Health, a prison governor, two prison GPs, a health protection specialist, a prison healthcare manager, a pharmaceutical adviser, a researcher, and two lay members.

Both guidelines were developed following standard NICE guideline methodology.[5]

A scope was produced which specified what the guideline would and would not cover. The GC developed clinical questions and review protocols for each question, specifying the search strategy (for both clinical and economic evidence) and method of evidence synthesis. GRADE methodology[6] was used to critically appraise the quality of the outcomes for each included study. GRADE quality ratings relate to the quality of the available evidence for assessed outcomes rather than the quality of the clinical study. The cost effectiveness of proposed interventions was evaluated where possible. The GC agreed recommendations for clinical practice based on the available evidence. Research recommendations were also agreed in areas of limited evidence. For the mental health of adults in contact with the criminal justice system guideline, the GC also used informal consensus methods to agree recommendations, when evidence was not found. Formal consensus was via the modified nominal group technique[7] — chosen because of its suitability within the guideline development process.

The draft scope and draft guideline were subject to a rigorous reviewing process in which stakeholder organisations were invited to comment. All comments received were responded to individually, and the respective GCs took these comments into consideration when producing the final versions of the scope and guideline.

NICE will conduct a review after publication of the guidance, to determine whether or not the evidence base has progressed significantly enough to alter the current guideline recommendations and require an update.

Other details

Three different versions of these guidelines have been produced: a full version containing all the evidence, the process undertaken to develop the recommendations, and all the recommendations, known as the "full guideline"; a short version containing a list of all the recommendations, known as the "short guideline"; and a version on the information for patients product, known as the "information for the public guideline." All of these versions are available from the NICE website (URLs TO BE INSERTED)

Box End

Contributors: All authors were involved in the conception and design of the work. All authors contributed to the acquisition, analysis, and interpretation of data. All authors reviewed the initial draft, were involved in writing further drafts, reviewed and approved the final version for publication, and agreed to be accountable for all aspects of the article. RB is the guarantor.

Funding: The National Guideline Alliance and the National Guideline Centre were commissioned and funded by the National Institute for Health and Care Excellence to develop these guidelines. BAJP and HT are employees of the National Guideline Centre, which is commissioned and funded by NICE to develop guidelines. 
Competing interests: We declare the following interests based on NICE's policy on conflicts of interests (available at www.nice.org.uk/Media/Default/About/Who-we-are/Policies-andprocedures/code-of-practice-for-declaring-and-managing-conflicts-of-interest.pdf): NK is the author of a (draft) book on the problem with diagnoses and was appointed to a project group for Centre for Mental Health and Howard League for Penal Reform on reducing deaths in detention. The authors' full statements can be viewed at www.bmj.com/content/bmj/356/bmj.j1378/related\#datasupp.

1. Ministry of Justice. Offender management statistics quarterly, England and Wales, July to September 2016 (with prison population as at 31 December 2016). 2017. www.gov.uk/government/uploads/system/uploads/attachment_data/file/585870/omsqbulletin-q3-2016.pdf.

2. National Institute for Health and Care Excellence. Physical health of people in prison [ NG57]. NICE, 2016. https://www.nice.org.uk/guidance/ng57

3. National Institute for Health and Care Excellence. Mental health of adults in contact with the criminal justice system [CG 66]. NICE, 2017.

https://www.nice.org.uk/guidance/ng66

4. National Institute for Health and Care Excellence. Medicines optimisation: the safe and effective use of medicines to enable the best possible outcomes [NG5]. NICE, 2015. https://www.nice.org.uk/guidance/ng5

5. National Institute for Health and Care Excellence. Developing NICE guidelines: the manual. NICE, 2016. www.nice.org.uk/process/pmg20/chapter/introduction-and-overview.

6. Guyatt GH, Oxman AD, Vist GE, et al; GRADE Working Group. GRADE: an emerging consensus on rating quality of evidence and strength of recommendations. $B M J$ 2008;336:924-6.

7. Bernstein SJ, Laouri M, Hilborne LH, et al. Coronary angiography: a literature review and ratings of appropriateness and necessity. RAND, 1992. 